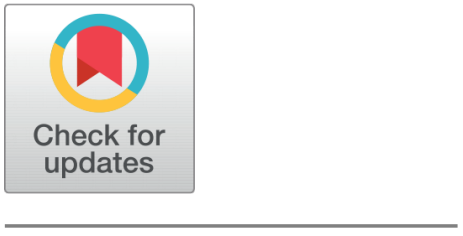

OPEN ACCESS

Received: 08.06.2021

Accepted: 20.07.2021

Published: 24.08 .2021

Citation: Singh PP (2021) A Novel Chaotic System with Wide Spectrum, its Synchronization, Circuit Design and Application to Secure Communication. Indian Journal of Science and Technology 14(28): 2351-2367. https://doi.org/ 10.17485/IJST/v14i28.1035

* Corresponding author. piyushpratapsingh@gmail.com

Funding: TEQIP-III Minor Research Grant, National Institute of Technology Meghalaya, India. Sanctioned Project Ref. No. NITMGH/TEQIP-III/MP/2019-20/269

Competing Interests: None

Copyright: @ 2021 Singh. This is an open access article distributed under the terms of the Creative Commons Attribution License, which permits unrestricted use, distribution, and reproduction in any medium, provided the original author and source are credited.

Published By Indian Society for Education and Environment (iSee)

ISSN

Print: 0974-6846

Electronic: 0974-5645

\section{A Novel Chaotic System with Wide Spectrum, its Synchronization, Circuit Design and Application to Secure Communication}

\author{
Piyush Pratap Singh ${ }^{1 *}$ \\ 1 Department of Electrical Engineering, NIT Meghalaya, Laitumkhrah, Shillong, 793003, \\ Meghalaya, India
}

\section{Abstract}

Objective: To investigate a novel chaotic system with unique features, its synchronization using nonlinear active control, analog circuit design and application to secure communication. Methods/Analysis: Dynamical tools such as dissipative analysis, instability of equilibrium points, sensitivity to initial conditions, 0-1 test, recurrence plot, Poincare map, Lyapunov exponents, Lyapunov dimension, frequency spectrum and basin of attraction. Synchronization is achieved using modified nonlinear active control technique and analog circuit design, implementation is done in NI Multisim platform. MATLAB and Multisim results are presented to meet the adequate verification of theoretical approach. Findings: A three-dimensional chaotic system with only two nonlinear terms, three parameters and a total of eight terms are proposed. The proposed system has three saddle focus type equilibria. The proposed system is topologically different from Lorenz's and Rossler's, Lu's, Chen's, and Liu's families. Such dynamic systems are very few in the literature as per authors best knowledge. The system has basin of chaotic attractors for which first Lyapunov exponent ranges between 2.5 to 3 . Frequency spectrum and large positive Lyapunov exponent result comparatively large bandwidth of the proposed systems against some well-known chaotic systems. Chaos, periodic and stable behaviors are obtained by altering the system parameters. Novelty/Application: The proposed three-dimensional chaotic system has significant chaotic behavior and broader spectrum than the six chaotic systems like Lorenz, Rossler, Lu, Chen, BG and Liu systems. Unlike the conventional active control approach, the proposed nonlinear active control does not result decoupled error dynamics. The system has significantly large bandwidth which is helpful in the masking of message signals and enhances the security of transmitted signals during communication.

Keywords: Analog circuit design; Chaos; Chaotic system; Chaosbased communication; Chaos synchronization; Nonlinear active control 


\section{Introduction}

Past three decades, chaotic phenomena, synchronization, and control of chaos behavior have been found and investigated in engineering and technology. A chaotic system has complex nonlinear behaviors and characteristics like sensitivity to initial conditions, long-term aperiodic behavior, and a wideband spectrum of Fourier transform ${ }^{(1)}$. Such features are observed in the real system ${ }^{(2)}$, Chemical reactor system ${ }^{(3)}$, Ecological system ${ }^{(4)}$, Communication system ${ }^{(5)}$ and Complex Network ${ }^{(6)}$, etc. Several novel chaotic systems have been reported and designed to date, given the first three-dimensional (3D) autonomous chaotic system reported by Lorenz ${ }^{(7)}$, such as Rossler system ${ }^{(8)}$, Chen system $^{(9)}$, Chua system $^{(10)}$, Liu system $^{(11)}$, Lu system $^{(12)}$, other novel chaotic systems ${ }^{(13-21)}$, etc. Further, design of novel chaotic systems and their electronic circuit implementation are still open and attracting attention due to application like communication and information processing ${ }^{(22,23)}$, etc.

Communication security is a crucial problem nowadays. Chaotic system synchronization helps toward improvement in the application of secure communication. Simultaneously, the reported chaotic systems have disadvantages during communication security, and the possible reasons are (1) the value of first Lyapunov Exponent (LE) is small so that the disordered degree of chaotic signal is not high. (2) the bandwidth of the chaotic signals is very low. First LE of a dissipative dynamic gives quantitative measure as positive nature of first LE implies chaos, and its value reflects disorder in chaos behavior. The bandwidth of wellknown Lorenz, Rossler, Lu, Chen, and Liu chaotic signals ranges between 2-9 Hz approximately and needs further improvement in the context of applications like information and voice and Laser communications.

Very first time Pecora \& Carroll ${ }^{(24)}$ investigated the synchronization between chaotic systems and illustrated that any chaotic dynamics could be synchronized with different initial conditions. Owing to this fact, various control schemes have been reported to achieve chaotic systems synchronization and control till date such as nonlinear active control ${ }^{(25)}$, adaptive control ${ }^{(26)}$, backstepping control ${ }^{(27)}$, sampled-data feedback method ${ }^{(28)}$, time-delay feedback method ${ }^{(29)}$, sliding mode control ${ }^{(30)}$, passive control $^{(31)}$, optimal control ${ }^{(32)}$, Fuzzy control ${ }^{(33)}$, PID control ${ }^{(34)}$, etc. The above control techniques have disadvantages like they result decoupled error dynamics which are unlike and trivial and have been studied by several researchers.

The above literature has some crucial issues and research gaps which are required to be explore in view of different applications. The major issues are identified as (1) there is a need to develop a novel chaotic system with higher positive LE and broader bandwidth and (2) A improved nonlinear control technique is required which does not result unlike, trivial, decoupled error dynamics. Motivated by the above issues and research gaps, following objectives are decided for this work.

1. To investigate an autonomous chaotic system with large LE and wideband frequency spectrum.

2. To design a nonlinear active control technique which results coupled error dynamics for the synchronization of chaotic systems and its application.

3. Analog circuit design and implementation in NI Multisim platform.

Therefore, a 3D autonomous, dissipative chaotic system is proposed, which has a more chaotic and broader spectrum than six well-known existing chaotic systems. Different dynamical properties of the system are analyzed for effective verification of chaotic behavior. The frequency spectrum is analyzed and compared with well-known chaotic systems and shows that the proposed system has comparatively large bandwidth. The modified nonlinear active control is proposed which results coupled error dynamics. Further, synchronization between proposed chaotic system and its application to secure communication explored using a novel nonlinear active control followed by analog circuit design and simulation in NI Multisim platform.

The rest of the paper is arranged as: the introduction and dynamical behaviors of the proposed novel chaotic system are presented in Section 2. In Section 3, different dynamical behaviors of the proposed novel system with variations of different parameters are analyzed. In Section 4, synchronization of the proposed novel chaotic system using nonlinear active control technique is discussed. The analog circuit is designed for proposed chaotic system and its synchronization and simulation using NI Multisim are presented in Section 5. In Section 6, application to secure communication is explored. Finally, conclusions and future scopes are summarized and listed in Section 7.

\section{Chaotic System Description}

The dynamics of proposed 3D autonomous system is described as:

$$
\left\{\begin{array}{l}
\dot{x}_{1}=a x_{1}-x_{2}^{2} \\
\dot{x}_{2}=b\left(x_{3}-x_{2}\right) \\
\dot{x}_{3}=(c-b) x_{2}+c x_{3}+x_{1} x_{2}
\end{array}\right.
$$


Where $x_{1}, x_{2}, x_{3}$ are state variables, and $a, b, c$ are real and constant parameters. System (1) has chaotic behavior for parameters $a=-10, b=55, c=37$. Different properties of proposed system (1) are analyzed further to validate different dynamical behaviors.

\subsection{Dissipative analysis}

The divergence $(\operatorname{div} V)$ of the proposed dynamics (1) is obtained as:

$$
\begin{aligned}
\operatorname{div} V & =\frac{\partial \dot{x}_{1}}{\partial x_{1}}+\frac{\partial \dot{x}_{2}}{\partial x_{2}}+\frac{\partial \dot{x}_{3}}{\partial x_{3}} \\
& =a-b+c<0
\end{aligned}
$$

Since $a-b+c<0$, the system dynamics (1) is dissipative with exponentially decay behavior as:

$$
\frac{d V}{d t}=V_{0} e^{(a-b+c) t}
$$

It means that the phase space behavior of system exponential converses to zero as $t \rightarrow \infty$ at the rate $(a-b+c)$.

\subsection{Equilibria analysis}

The equilibria of the proposed system (1) are obtained by equating $\dot{x}_{1}=0, \dot{x}_{2}=0, \dot{x}_{3}=0$. Three equilibria are given as

$$
\left\{\begin{array}{l}
E_{1}=[0,0,0]^{T} \\
E_{2}=[-(2 c-b), \sqrt{a(b-2 c)}, \sqrt{a(b-2 c)}]^{T} \\
E_{3}=[-(2 c-b),-\sqrt{a(b-2 c)},-\sqrt{a(b-2 c)}]^{T}
\end{array}\right.
$$

At $E_{1}=\left[\begin{array}{lll}0,0 & 0\end{array}\right]^{T}$, Jacobian linearization of the system (1) is achieved as:

$$
J_{E_{1}}=\left[\begin{array}{ccc}
a & -2 x_{2} & 0 \\
0 & -b & b \\
x_{2} & \left(c-b+x_{1}\right) & c
\end{array}\right]_{E_{1}}=\left[\begin{array}{ccc}
-10 & 0 & 0 \\
0 & -55 & 55 \\
0 & -18 & 37
\end{array}\right]
$$

Eigenvalues are calculated as $\lambda_{1}=24.5559, \lambda_{2}=-42.5559$, and $\lambda_{3}=-10$. Since $\lambda_{1}>0$ and $\lambda_{2}, \lambda_{3}<0$, the $E_{1}$ equilibrium point has a saddle point behavior.

Similarly, at the equilibrium point $E_{2}$, eigenvalues are calculated as $\lambda_{1,2}=4.9198 \pm 22.9809 i$, and $\lambda_{3}=-37.8396$ which exclaim that an unstable saddle-focus nature exists about equilibrium point $E_{2}$. Equilibrium point $E_{3}$ has an unstable saddlefocus behavior due to symmetricity between $E_{2}$ and $E_{3}$. It is concluded that the system has three unstable equilibria with saddle focus and point behaviors.

\subsection{Sensitivity to initial conditions}

Chaotic systems are susceptible to initial conditions, i.e., a slight change in initial conditions may result in significantly diverse output in the state behaviors. Two very close initial conditions are considered as $\left[\begin{array}{lll}-4.1 & 4.1 & 2.5\end{array}\right]^{T}$ and $\left[\begin{array}{lll}-4.10001 & 4.10001 & 2.50001\end{array}\right]^{T}$. The change in the initial condition is made at the fifth decimal place and simulation result shown in Figure 1 reveals that two behaviors excited at different initial conditions are identical till $t=4$ seconds and after that the chaotic behaviors are quite different from each other. 

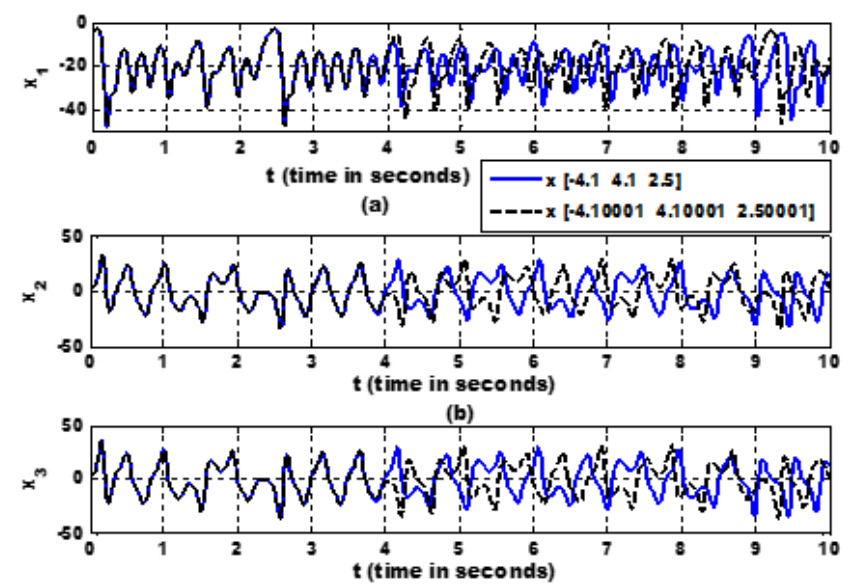

(c)

Fig 1. Shows sensitivity to initial conditions. There are abrupt changesin time responses of $x(t)$ after 4 second with small change in initial conditions.

\subsection{Analysis of 0-1 test}

It is a binary test to detect different behaviors of a system ${ }^{(35)}$. The chaotic and periodic behaviors are defined on the $k_{c}$ value. If $k_{c} \approx 1$ then the behavior is chaotic and $k_{c} \approx 0$ then the behavior is periodic. Here, $0-1$ test for $x_{2}(t)$ signal of the system is presented in Figure 2. Figure 2 shows (a) the Brownian like nature, (b) asymptotic growth rate $\left(k_{c}\right)$, and (c) mean square displacement $M_{c}(n)$. We have $k_{c}=0.9944 \approx 1$ which confirms chaotic behavior of system (1) for $a=-10, b=55, c=37$.
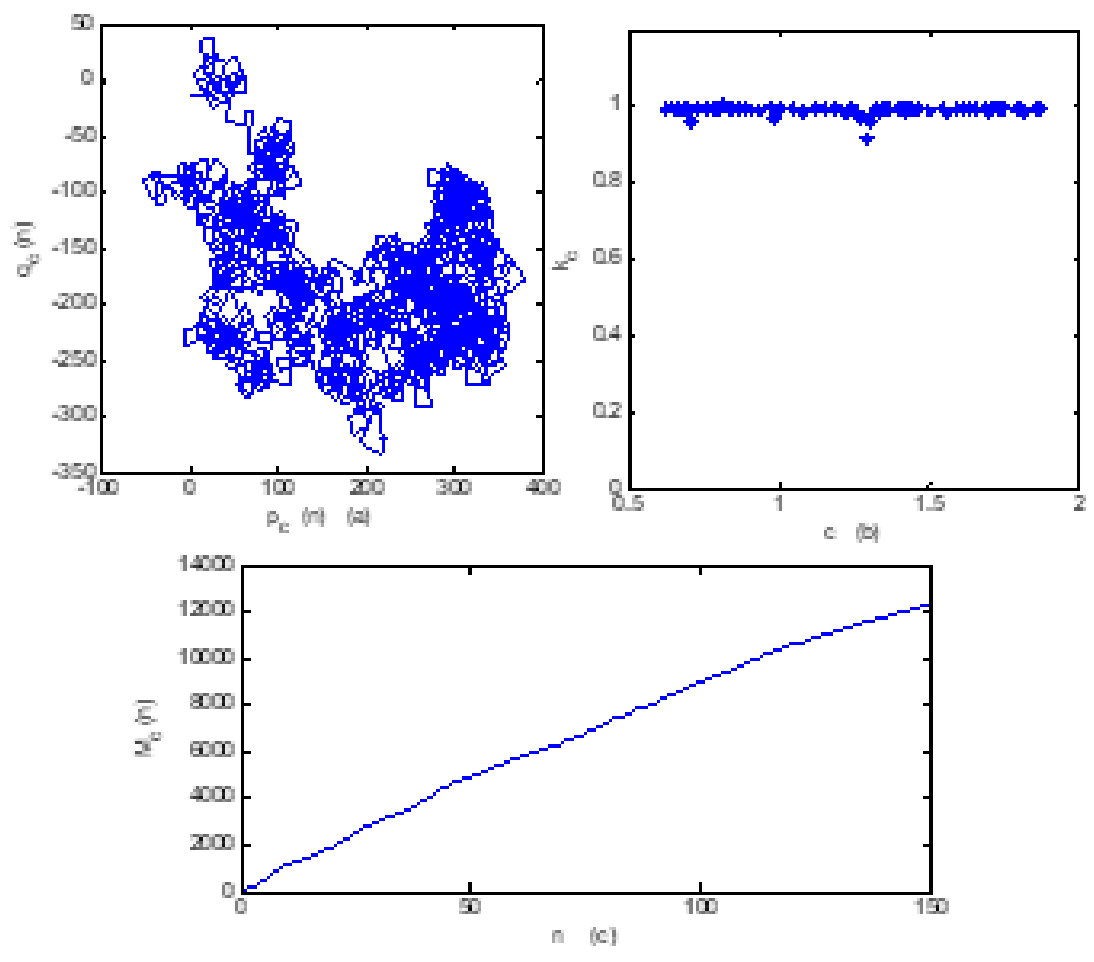

Fig 2. 0 - 1 test of the $x_{2}$ signal of the new system with $a=-10, b=55, c=37, x(0)=(0,0,0)^{T}, \triangle t=1:(a)$ dynamics of the translation components $\left[p_{c}(n), q_{c}(n)\right]$, (b) asymptotic growth rate $k_{c}$ and (c) mean square displacement $M_{c}(n)$. 


\subsection{Recurrence plot}

A recurrence plot is used to study phase space behaviours of the higher dimensional system. It envisions the recurrence of states variables in phase plane ${ }^{(36,37)}$ :

$$
R_{i, j}=\odot\left(\left\|x_{i}-x_{j}\right\|\right), x_{i} \in R^{m}, i, j=1 \ldots N
$$

A recurrence is a distance between $i$ and $j$ points $\in \varepsilon$ and pairwise test to find first characterization of data. It is a $N \times N$ matrix which generates black and white dots where black dots represent recurrence of data and used to find the recurrence of the state of the novel system (1). We chose the resampled first state of the proposed system and constructed a phase space trajectory with time delay of 0.34 seconds. Length of original data series is taken 600 and the length of data series for obtained phase space trajectory is 596. The recurrence of the $x_{1}$ states of the system are shown in Figure 3.

In Figure 3, the short diagonal line represents the epochs. The distance between obtained diagonals represents the periods of cycles either differs, constant or both. Thus, Figure 3 validates the complex chaotic nature of the new chaotic system (1).

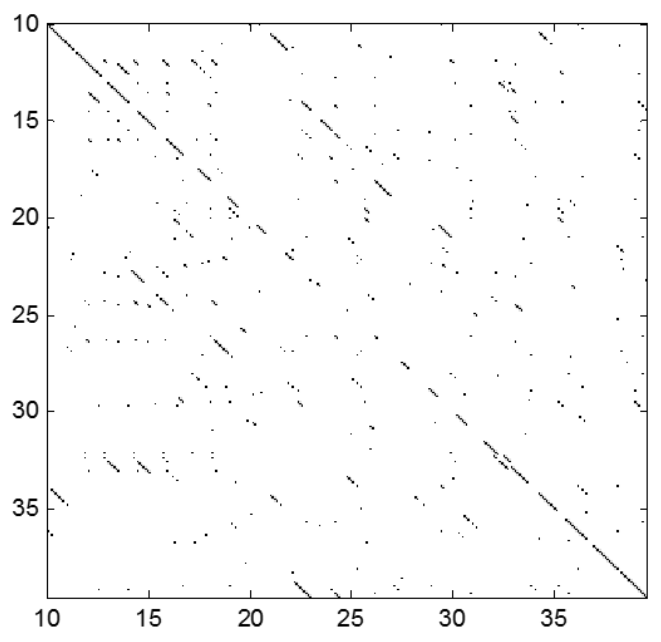

Fig 3. The Recurrence plot of the system (1) for $a=-10, b=55, c=37$ constructed using a time delay of 0.34 with embedding dimension of $D_{E}=3$.

\subsection{Poincare map of the novel chaotic system}

Poincare map is a tool to measure the presence of chaos in a dynamic system. Poincare maps of the system are plotted in the different planes for $x_{2}=0$ and $x_{3}=0$ and are shown in Figure 4 confirm that the system has chaotic behaviour.
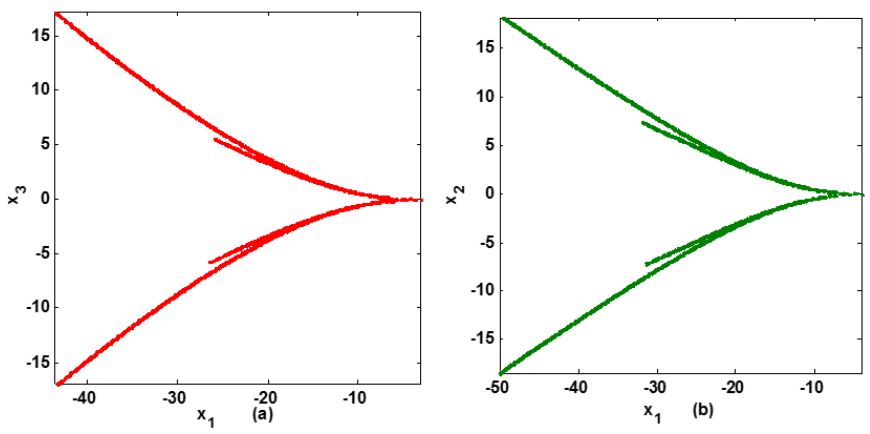

Fig 4. The Poincare map of the chaotic system (1) for $a=-10, b=55, c=37:$ (a) in $x_{1}-x_{3}$ plane for $x_{2}=0$, (b) in $x_{1}-x_{2}$ plane for $x_{3}=0$. 


\subsection{Lyapunov exponents and Lyapunov dimension}

The Lyapunov exponents of the proposed system (1) are calculated for the verification of chaotic behavior. Figure 5 shows the time evolution of the Lyapunov exponents of the system (1). As shown in Figure 5, the Lyapunov exponents are $L_{1}=2.9732$, $L_{2}=-0.00805 \approx 0, L_{3}=-30.9649$. The sign of LEs as $[+, 0,-]$ signifies chaotic behavior.

Lyapunov dimension of the chaotic system is obtained as:

$$
L_{D}=2+\frac{1}{\left|L_{3}\right|} \sum_{i=1}^{2} L_{i}=2+\frac{2.9652}{30.9649}=2.0957
$$

The fractional value of Lyapunov dimension confirms chaotic behavior.

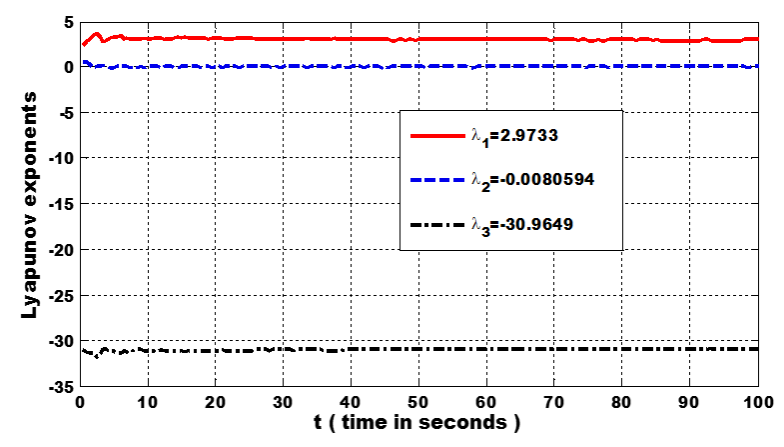

Fig 5. Dynamical behavior of Lyapunov exponents (LEs).

\subsection{Frequency spectrum analysis}

Chaotic signal has a noise-like behavior and thus there is an intuitive feeling that it has a broad range of frequency spectrum or large bandwidth. But unfortunately, message signal extraction has been reported. One of the reasons is that the bandwidth of the chaotic signal is low ${ }^{(15)}$. Therefore, chaotic system with high bandwidth is the critical need of secure communication applications. The bandwidth of proposed system is approximately $24 \mathrm{~Hz}$ as shown in Figure 6, which is relatively more significant than the bandwidth of widespread chaotic systems namely Lorenz ${ }^{(10)}$, Rossler ${ }^{(10)}$, Chen ${ }^{(38)}, \mathrm{Lu}^{(25)}, \mathrm{BG}^{(25)}$ and Liu ${ }^{(39)}$ systems (considering 20\% cut-off). A comparison of proposed chaotic system among different chaotic systems based on LEs, Lyapunov dimension, and bandwidth is done and summarized in Table 1 . To decide the bandwidth, $20 \%$ cutoff is considered as suggested in the literature. Large value of the first LE and wide bandwidth reflect the importance and novelty of the proposed chaotic system.

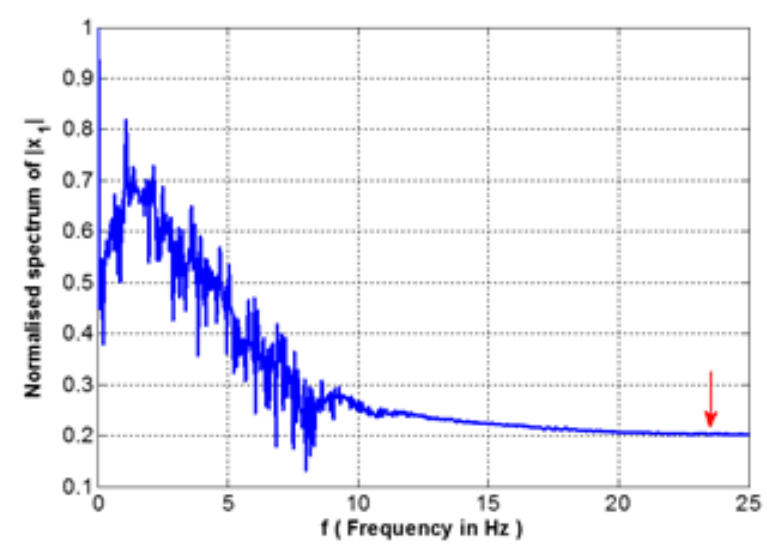

Fig 6. Shows the normalised frequency spectrum of absolute values of the $x_{1}(t)$ chaotic signal. 
Table 1. Comparison among different chaotic systems

\begin{tabular}{llll}
\hline Chaotic System & Lyapunov Exponents & Lyapunov Dimension & Bandwidth \\
\hline Rossler [10] & $0.0714,0,-5.3943$ & 2.013 & $2.5 \mathrm{~Hz}$ \\
Lorenz [10] & $0.0906,0,-14.572$ & 2.062 & $2.6 \mathrm{~Hz}$ \\
Lu [25] & $1.5046,0,-22.5044$ & 2.066 & $3.0 \mathrm{~Hz}$ \\
BG [25] & $0.9694,-0.008,-14.6452$ & 2.065 & $2.1 \mathrm{~Hz}$ \\
Liu [39] & $1.6432,0,-14.42$ & 2.113 & $5.0 \mathrm{~Hz}$ \\
Chen [38] & $1.997,0.002,-11.9943$ & 2.166 & $8.8 \mathrm{~Hz}$ \\
Proposed system & $2.9733,-0.008,-30.9649$ & 2.096 & $24 \mathrm{~Hz}$ \\
\hline
\end{tabular}

In the next Section, parameters are varied, and different behaviors of the proposed system are observed by Lyapunov spectrum plots and basin of attraction to show various dynamical behaviors.

\section{Complex Behaviors of Proposed Chaotic System}

\subsection{Lyapunov spectrum}

In this section, the parameter $a$ is varied in the interval $-54 \leq a \leq 0$ while $b=55$ and $c=37$ are constant. The Lyapunov spectrum of system (1) with variation of parameter $a$ is shown in Figure 9(a). It is observed that the nature of three Lyapunov exponents are $L_{1} \approx 0, L_{2}<0$ and $L_{3}<0$ when $-54 \leq a<-14.6$, and $-1.4 \leq a<0$. Therefore, system (1) has periodic behaviors in these intervals. The phase plane behaviors of the system (1) by considering an arbitrary value of parameter $a$ from the above two breaks are shown in Figure 7. The LEs with parameters $a=-21, b=55, c=37$ are $L_{1}=0.0065 \approx 0, L_{2}=$ -1.4690 and $L_{3}=-37.5373$ which confirm periodic nature of proposed system (1).

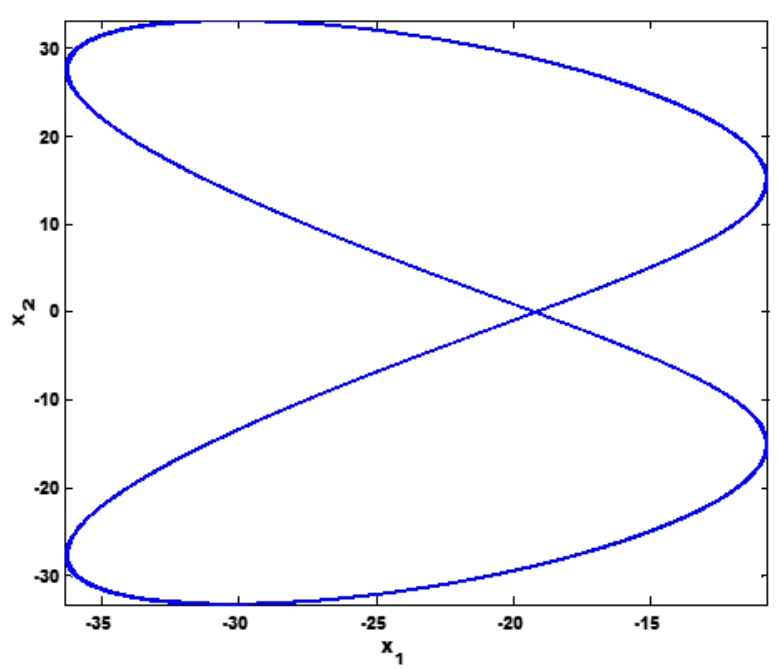

(a) when $\mathrm{a}=-21, \mathrm{~b}=55$, and $\mathrm{c}=37$

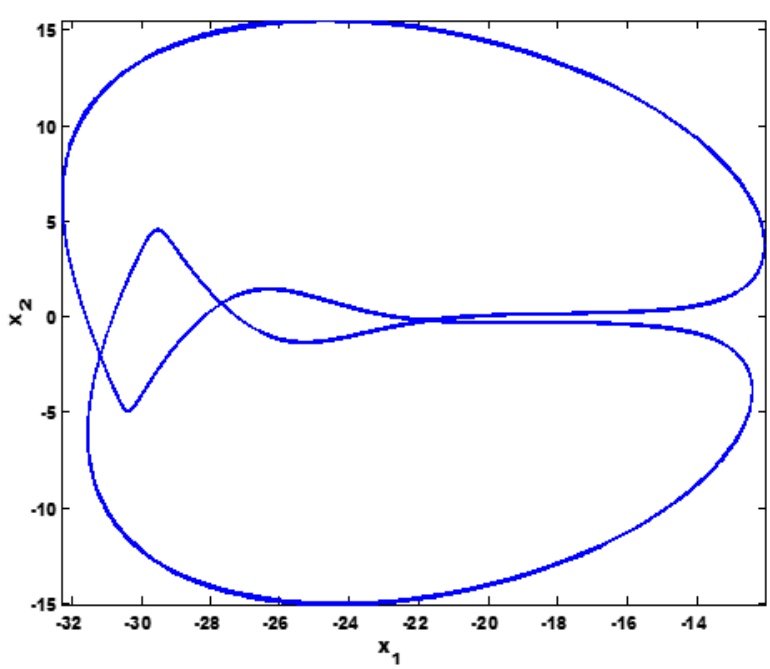

(b) when $a=-1.2, b=55$, and $c=37$

Fig 7. Phase plane behavior of the system in (a)-(b) with different value of the parameter $a$.

Further, when $-14.6 \leq a<-1.4$, system (1) has three LEs as $L_{1}>0, L_{2} \approx 0$ and $L_{3}<0$ signify chaotic behavior. The phase plane behavior of the system (1) with parameters $a=-8, b=55$, and $c=37$ is shown in Figure 8 which confirms chaotic nature. The LEs with same set of parameters are calculated as $L_{1}=3.473324, L_{2}=-0.0046 \approx 0, L_{3}=-29.468313$ which also validate the chaotic behavior of system (1). 


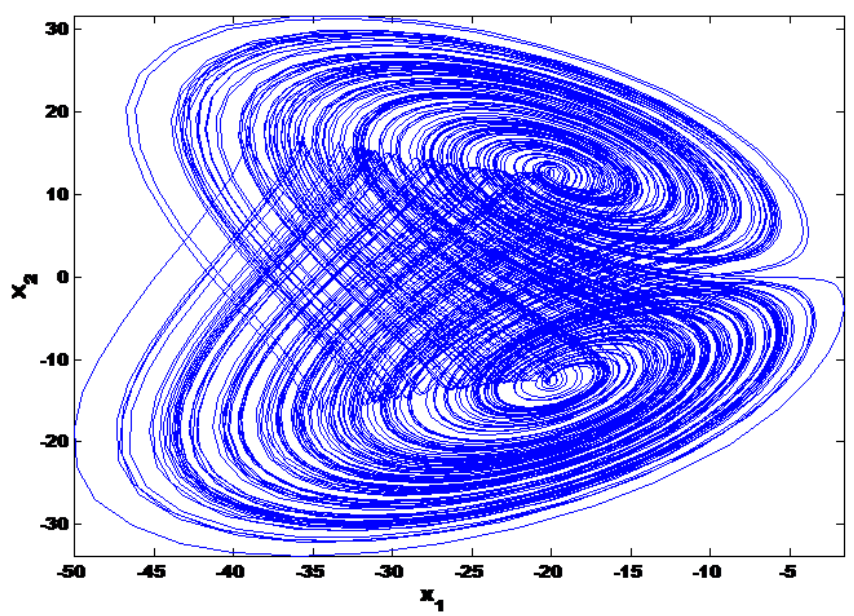

Fig 8. Phase plane behavior shows chaotic nature of system with $a=-8, b=55$ and $c=37$.

Similarly, LEs by varying parameter $b$ and parameters $c$ are shown in Figure 9(b) and 9(c), respectively, where parameters $b$ and $c$ are varied in the interval $40 \leq b \leq 66$ and $30 \leq c \leq 52$, respectively, while keeping other parameters fixed. Different dynamical behaviors of system (1) with the variation of $a, b$ and $c$ parameters are listed in Table 2 .
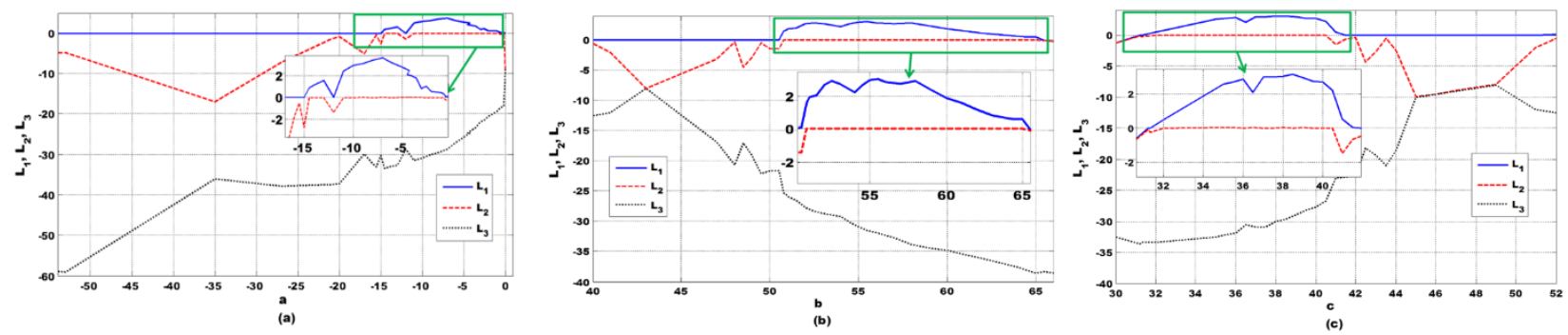

Fig 9. Lyapunov spectrums with respect to variation of a, b, c parameters in (a)-(c).

Table 2. Dynamical behaviors with the variation of $a, b$, and c parameters

\begin{tabular}{lll}
\hline Varying Parameter & Range & Dynamical behavior \\
\hline \multirow{4}{*}{$a$} & $a<-14.6$ & Periodic (Limit cycle) \\
& $-14.6 \leq a<-1.4$ & Chaos \\
& $-1.4 \leq a<0$ & Periodic (Limit cycle) \\
& $b<39.9$ & Stable \\
$b$ & $39.9 \leq b<50.7$ & Periodic (Limit cycle) \\
& $50.7 \leq b<65.3$ & Chaos \\
& $65.3 \leq b<66$ & Periodic (Limit cycle) \\
& $66 \leq b$ & Stable \\
& $30 \leq c<31.2$ & Stable \\
& $31.2 \leq c<31.9$ & Periodic (Limit cycle) \\
& $31.9 \leq c<41.3$ & Chaos \\
\end{tabular}




\subsection{Basin of attraction (sensitivity to initial conditions}

Bispectral plot with respect to initial condition is known as basin of attraction ${ }^{(40)}$. The sensitivity to initial conditions of the system (1) is shown in Figure 10 with respect first Lyapunov Exponent. Here, the $x_{1}=-4.10$ kept fixed and range of considered initial condition for $x_{2}$ and $x_{3}$ are varied as $-15<x_{2}<15$ and $-15<x_{3}<25$. It is apparent from Figure 10 that the system has chaotic behaviour with slight variation, irrespective of the range of initial conditions considered.

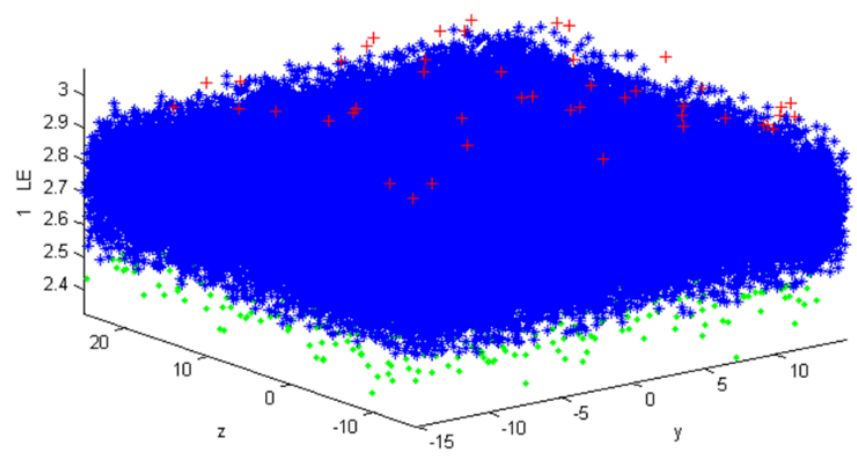

Fig 10. Basin of attraction of the chaotic system (1) with $a=-10, b=55, c=37$ where: red $(+)$ indicates $1 L E>3$, blue $(*)$ indicates $2.5<1 L E<3$, green (.) indicates $2.0<1 L E<2.5$.

\section{Synchronization of Novel Chaotic System}

The response chaotic system with added control inputs is given in (2).

$$
\left\{\begin{array}{l}
\dot{y}_{1}=a y_{1}-y_{2}^{2}+u_{1} \\
\dot{y}_{2}=b\left(y_{3}-y_{2}\right) \\
\dot{y}_{3}=(c-b) y_{2}+c y_{3}+y_{1} y_{2}+u_{2}
\end{array}\right.
$$

$y_{1}, y_{2}, y_{3}$ are state variables, and $a, b, c$ are real and constant parameters. $u_{1}, u_{2}$ are control inputs applied to the response system. The synchronization of drive-response systems is achieved for any initial conditions $x_{i}(0)$ and $y_{i}(0)$, if it satisfies (3).

$$
\lim _{t \rightarrow \infty}\|e(t)\|=\lim _{t \rightarrow \infty}\|y-x\|=0
$$

Let the error be defined as $e=y-x$ where $e \in R^{3}$. The designed nonlinear active control inputs are given in (4).

$$
\left\{\begin{array}{l}
u_{1}=y_{2}^{2}-x_{2}^{2} \\
u_{2}=x_{1} x_{2}-y_{1} y_{2}-k e_{3}
\end{array}\right.
$$

where $k$ is an external forcing, positive parameter.

Theorem. When $a=-10, b=55, c=37$ and $k>c+\frac{c^{2}}{4 b}$, systems (1) and (2) are synchronized globally using designed nonlinear active control laws (4).

Proof. The error dynamics is given as:

$$
\left\{\begin{array}{l}
\dot{e}_{1}=a e_{1} \\
\dot{e}_{2}=b\left(e_{3}-e_{2}\right) \\
\dot{e}_{3}=(c-b) e_{2}+(c-k) e_{3}
\end{array}\right.
$$

Using a Lyapunov function candidate as defined in (6).

$$
V(e)=\frac{1}{2}\left(e_{1}^{2}+e_{2}^{2}+e_{3}^{2}\right)
$$


$\dot{V}(e)=a e_{1}^{2}+c e_{2} e_{3}-b e_{2}^{2}-(k-c) e_{3}^{2}=-e^{T} P e$ where $e=\left[e_{1}, e_{2}, e_{3}\right]^{T}$, and a matrix is obtained as $P=\left[\begin{array}{ccc}-a & 0 & 0 \\ 0 & b & -\frac{c}{2} \\ 0 & -\frac{c}{2} & k-c\end{array}\right]$.

As per Theorem, $k>c+\frac{c^{2}}{4 b}$ so the matrix $P$ is positive definite. Therefore, response system (2) is globally asymptotically synchronized with drive system (1).

Remarks: The proposed control technique does not require the control law in all the dynamics. The designed nonlinear active control laws result nontrivial and coupled error dynamics.

The parameters are considered as $a=-10, b=55, c=37$ and the initial conditions of a drive (1) and response (2) systems are considered as $(-4.1,4.1,2.5]^{T}$ and $(6,7,8]^{T}$, respectively. Simulation results for synchronization between the drive and response systems and the designed control inputs are shown in Figures 11 and 12, respectively.

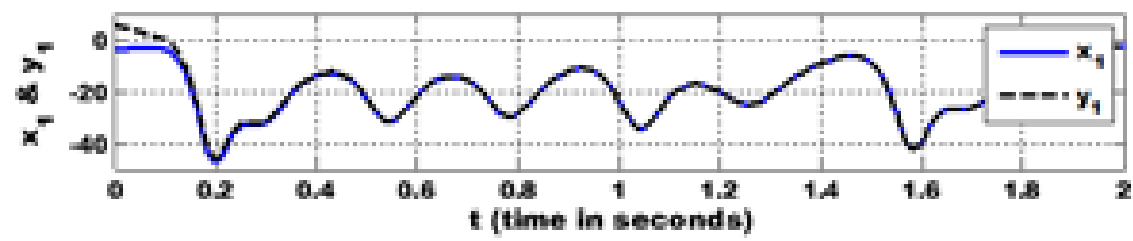

(a)

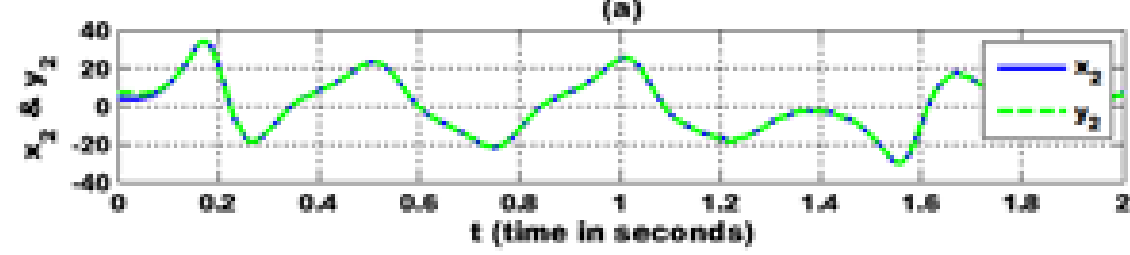

(b)

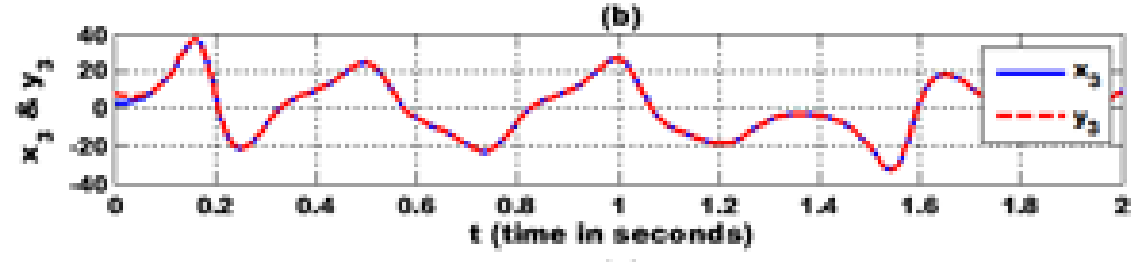

(c)

Fig 11. Synchronization of states between the drive and response systems.

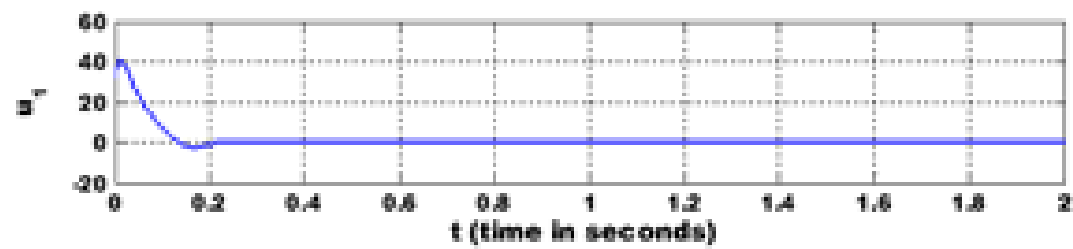

(a)

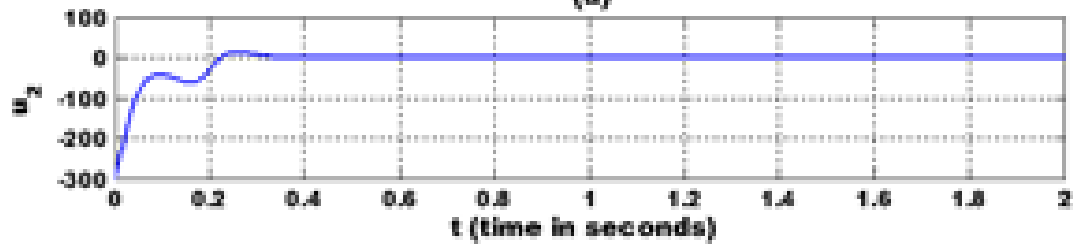

(b)

Fig 12. Control inputs to achieve the synchronization of the proposed chaotic system.

Synchronization error is shown in Figure 13. It is evident from Figures 11 and 13 that the synchronization of the proposed system is achieved successfully using designed control laws (4).

After the achievement of synchronization, design of analog circuit is discussed in the next section. 


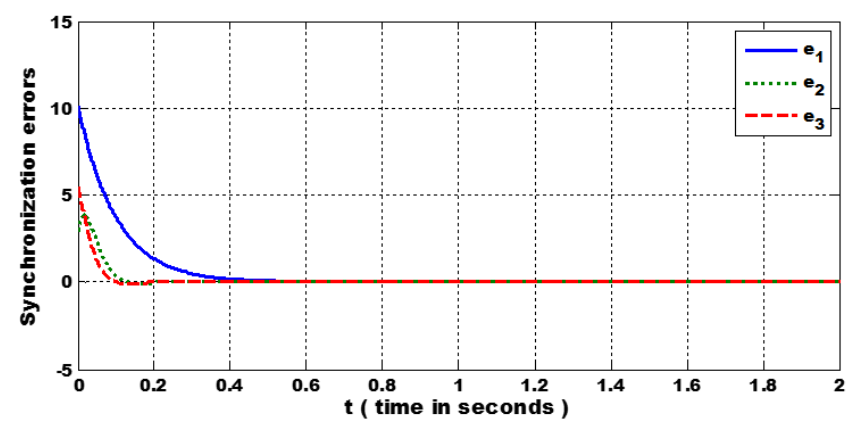

Fig 13. Synchronization errors between the drive (1) and response (2) chaotic systems.

\section{Analog Circuit Design for Synchronization of Novel Chaotic Systems}

Here, the proposed chaotic system (1) is designed as in (7) using analog components, i.e., resistances, capacitances, and Opamps of different values using NI Multisim.

$$
\left\{\begin{array}{l}
\frac{d x_{1}}{d t}=\frac{1}{R_{3} C_{2}}\left[-\left(\frac{R_{2}}{R_{19}}\right) x_{1}-\left(\frac{R_{2}}{R_{1}}\right) \frac{x_{2}^{2}}{10}\right] \\
\frac{d x_{2}}{d t}=\frac{1}{R_{9} C_{1}}\left[-\left(\frac{R_{11}}{R_{10}}\right) x_{2}+\left(\frac{R_{7}}{R_{6}}\right) x_{3}\right] \\
\frac{d x_{3}}{d t}=\frac{1}{R_{16} C_{3}}\left[\left(\frac{R_{15}}{R_{12}}\right) \frac{x_{1} x_{2}}{10}-\left(\frac{R_{18}}{R_{17}}\right) x_{3}+\left(\frac{R_{15}}{R_{13}}\right) x_{2}\right]
\end{array}\right.
$$

The analog circuit implementation is shown in Figure 14. The components values are given as: $C_{i}=100 n F(i=1,2,3) R_{i}=$ $10 \mathrm{k} \Omega$ for $(i=3,9,10,12,16), R_{1}=R_{19}=1 \mathrm{k} \Omega, R_{6}=R_{8}=1.818 \mathrm{k} \Omega, R_{7}=R_{15}=100 \mathrm{k} \Omega, R_{13}=5.558 \mathrm{k} \Omega, R_{14}=2.7027 \mathrm{k} \Omega$. Phase plane behaviors of the proposed chaotic system are shown in NI Multisim Oscilloscope as given in Figure 15.

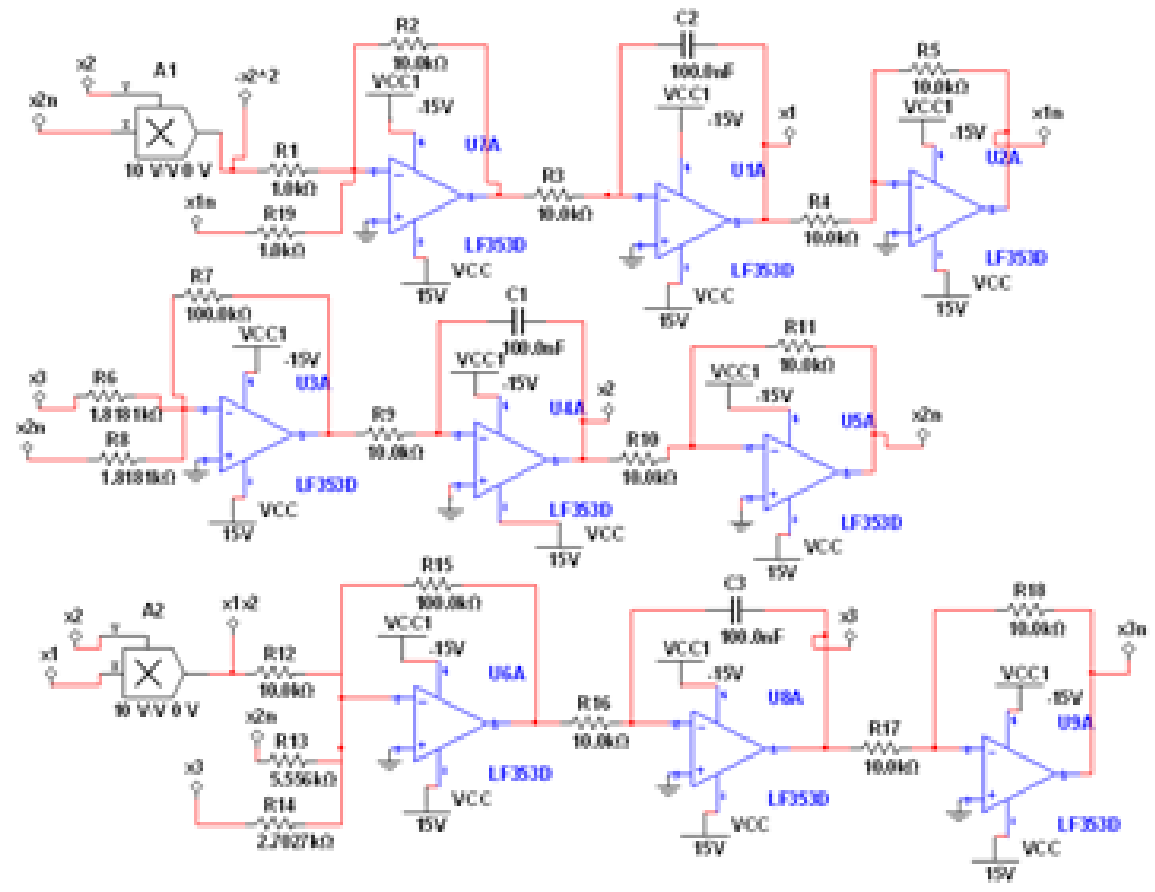

Fig 14. Analog circuit design of proposed novel chaotic system (1). 


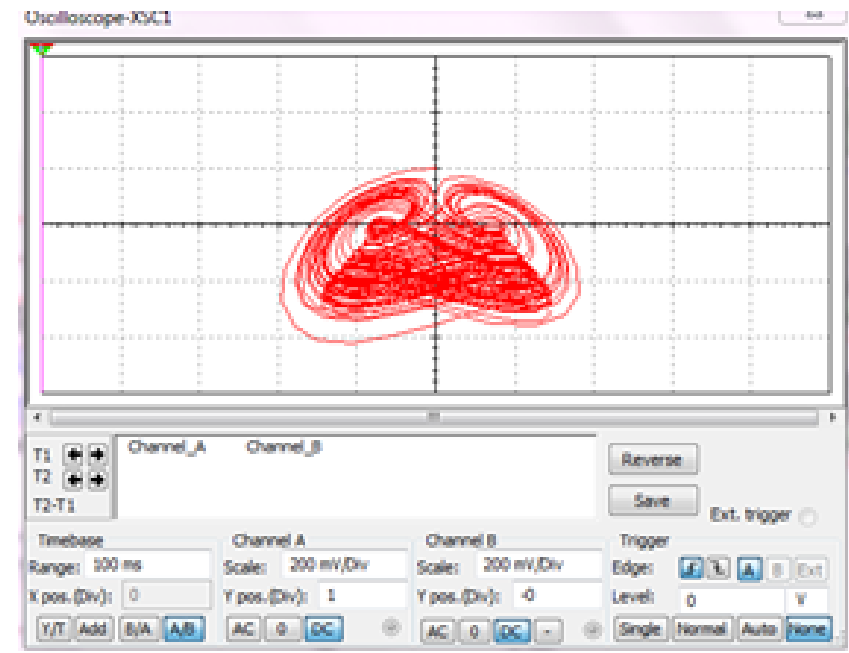

(a)

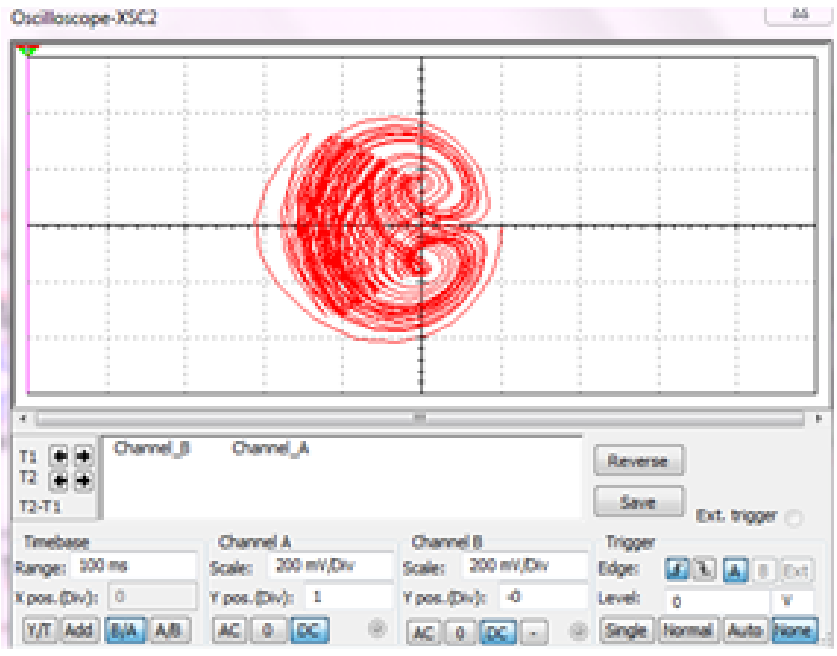

(b)

Fig 15. Shows phase plane behaviors of the system (1) in: (a) $x_{1}-x_{2}$ plane and (b) $x_{2}-x_{3}$ plane.

The response system dynamics (2) in terms of analog components are given in (8). The value of components are $C_{i}=100 \mathrm{nF}(i=4,5,6), R_{i}=10 \mathrm{k} \Omega$ for $i=22,28,31,35, R_{20}=R_{38}=1 \mathrm{k} \Omega, R_{25}=R_{27}=1.818 \mathrm{k} \Omega, R_{i}=$ $100 \mathrm{k} \Omega(i=21,26,34), R_{32}=5.558 \mathrm{k} \Omega, R_{33}=2.7027 \mathrm{k} \Omega$.

$$
\left\{\begin{array}{l}
\frac{d y_{1}}{d t}=\frac{1}{R_{22} C_{4}}\left[-\left(\frac{R_{21}}{R_{38}}\right) y_{1}-\left(\frac{R_{21}}{R_{20}}\right) \frac{y_{2}^{2}}{10}\right]+u_{1} \\
\frac{d y_{2}}{d t}=\frac{1}{R_{28} C_{5}}\left[-\left(\frac{R_{26}}{R_{27}}\right) y_{2}+\left(\frac{R_{26}}{R_{25}}\right) y_{3}\right] \\
\frac{d y_{3}}{d t}=\frac{1}{R_{35} C_{6}}\left[\left(\frac{R_{34}}{R_{31}}\right) \frac{y_{1} y_{2}}{10}+\left(\frac{R_{34}}{R_{33}}\right) y_{3}-\left(\frac{R_{34}}{R_{32}}\right) y_{2}\right]+u_{2}
\end{array}\right.
$$

The nonlinear active controller is given in (9) to achieve synchronization.

$$
\left\{\begin{array}{l}
u_{1}=\left(\frac{R_{21}}{R_{45}}\right) \frac{y_{2}^{2}}{10}-\left(\frac{R_{21}}{R_{46}}\right) \frac{x_{2}^{2}}{10} \\
u_{2}=-\left(\frac{R_{34}}{R_{49}}\right) e_{3}+\left(\frac{R_{34}}{R_{48}}\right) \frac{x_{1} x_{2}}{10}-\left(\frac{R_{34}}{R_{47}}\right) \frac{y_{1} y_{2}}{10}
\end{array}\right.
$$


where $R_{i}=10 \mathrm{k} \Omega(i=21,47,48), R_{34}=100 \mathrm{k} \Omega, R_{45}=R_{46}=1 \mathrm{k} \Omega, R_{49}=2.5 \mathrm{k} \Omega$. NI Multisim circuit implementation corresponding to the response system with the added control laws is shown in Figure 16. State response of drive and response systems and the synchronization errors are given in Figure 17 (a)-(c) and Figure 18, respectively.

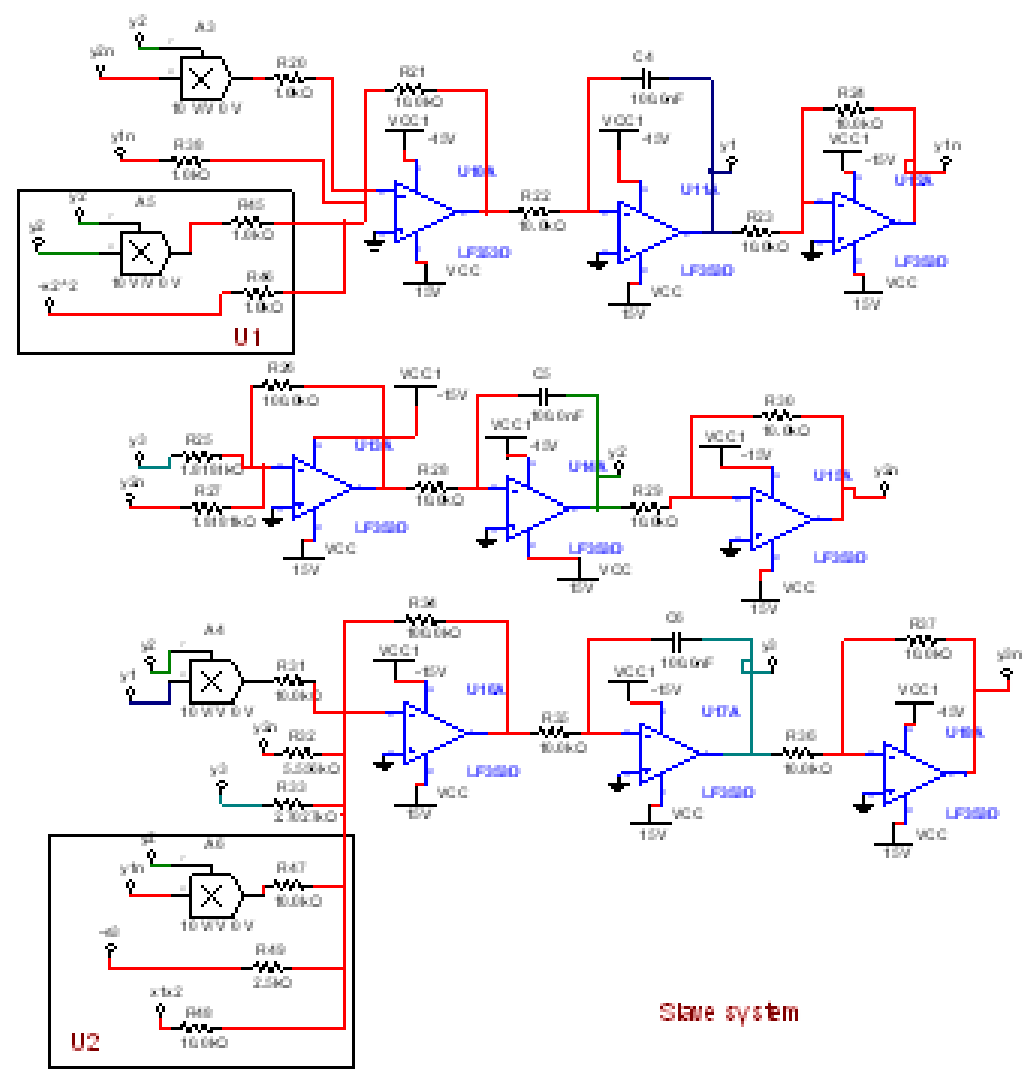

(a)

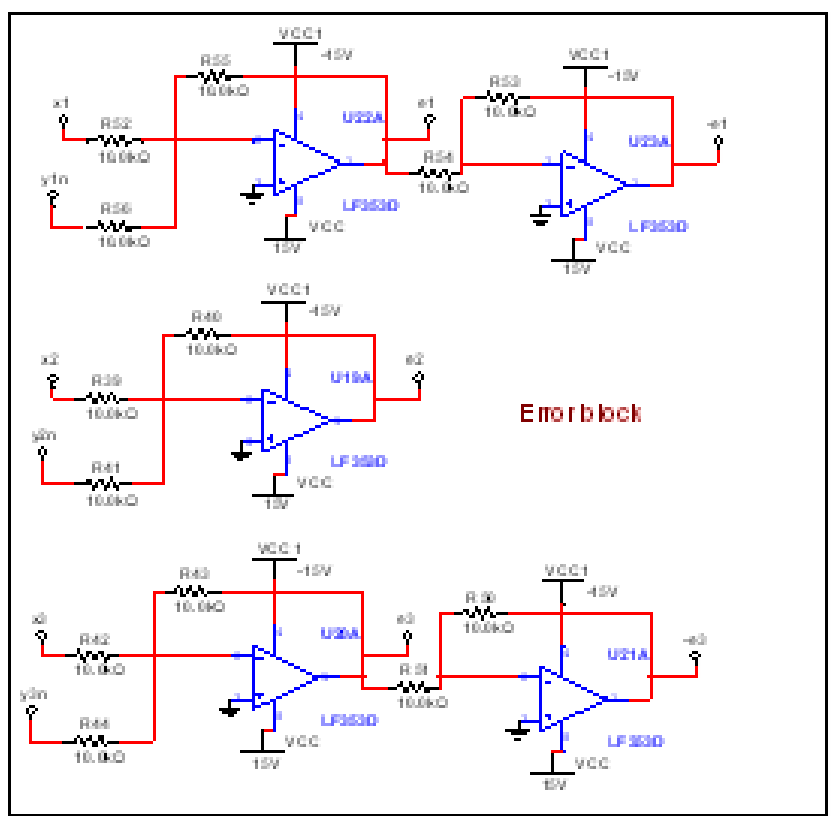

(b)

Fig 16. (a) Analog circuit design of the response system, and (b) shows the design of the error dynamics. 
Figures 17 and 18 validate the numerical simulation results and reveal that synchronization is achieved successfully. In the next Section, the application of the proposed system in secure communication is discussed.

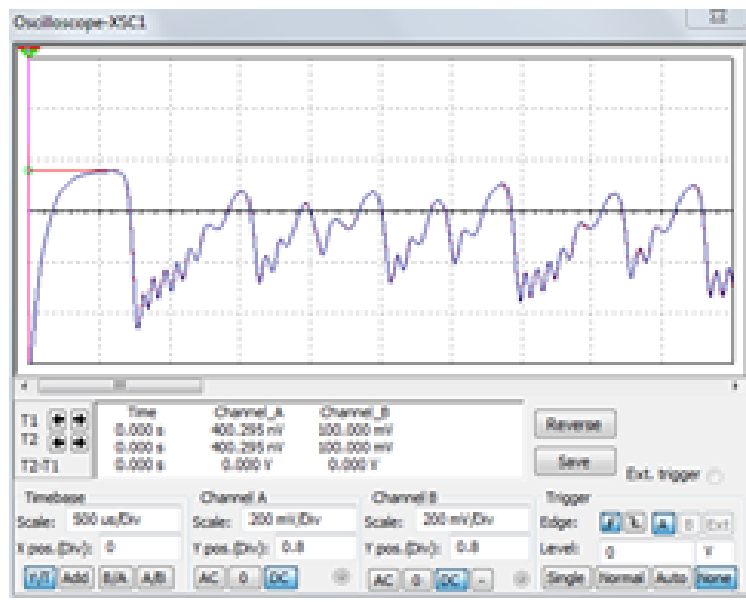

(a)

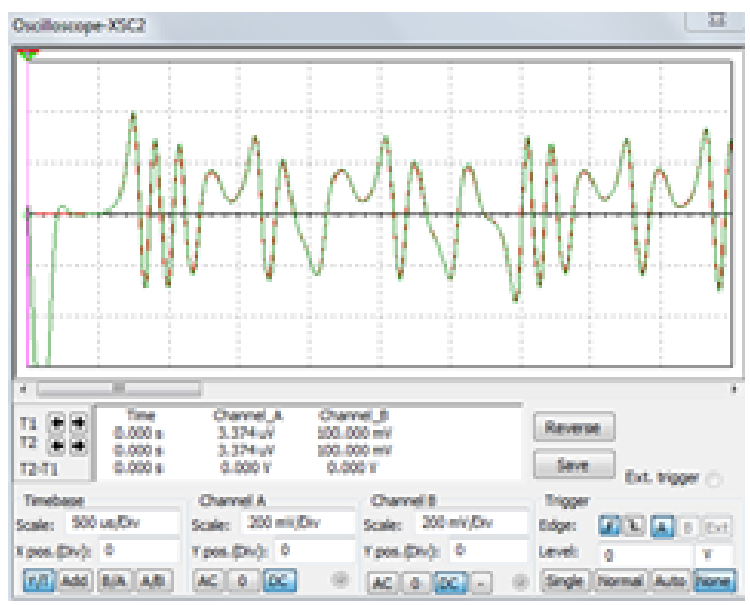

(b)

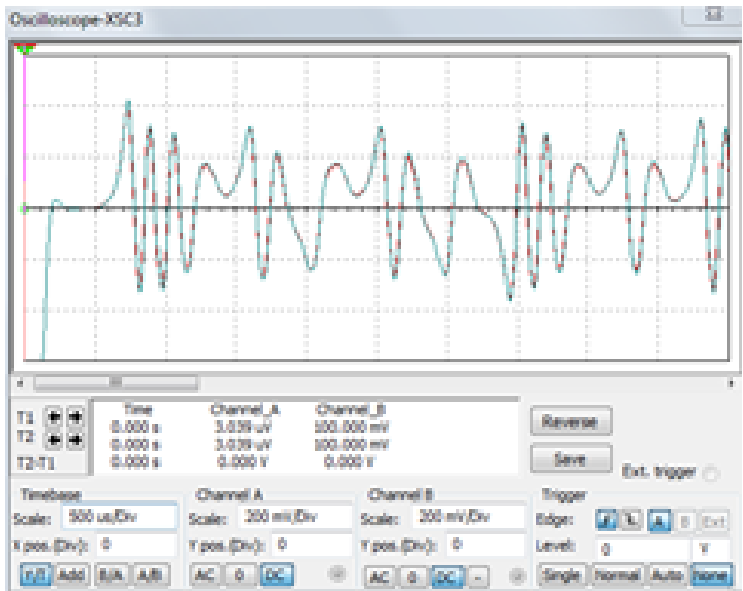

(c)

Fig 17. Synchronization between the states of the drive and response systems, (a) first states, (b) second states, (c) third states. 


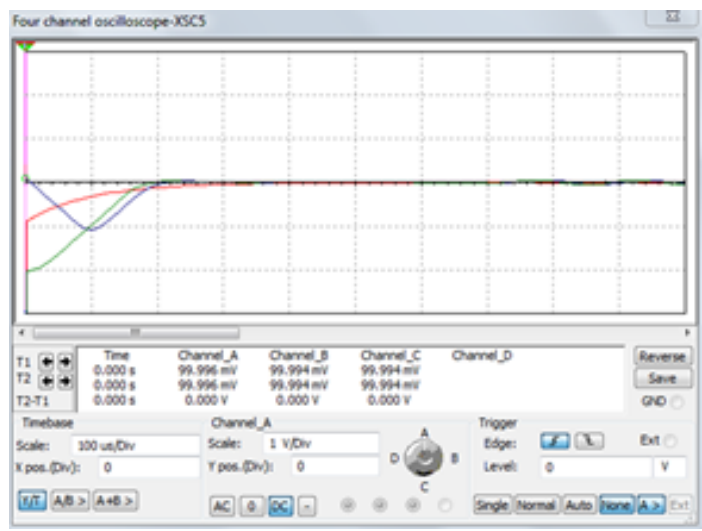

Fig 18. Synchronization errors where red line shows error $\left(e_{1}\right)$, blue $\left(e_{2}\right)$, and green $\left(e_{3}\right)$.

\section{Chaotic Secure Communication}

The potential use of synchronized chaotic systems is in communication and crystal laser ${ }^{(5,6)}$. The proposed communication setup using nonlinear active control technique is shown in Figure 19. The approach is based on the masking of message signal with a carrier signal (chaotic signal) at the transmitter end. Unmasking of message signal can be achieved, iff the synchronization between chaotic systems is achieved using appropriate nonlinear active control design at receiver end. A message signal $m(t)=5 \sin (200 \pi t)$ is added with $x_{3}$ state at the transmitter end and termed as transmitted signal $i_{T}=\left(x_{3}+m\right)$. At receiver end, the received message signal is recovered as $\widehat{m}(t)=i_{T}-y_{3}(t)$ synchronization is achieved using designed control.

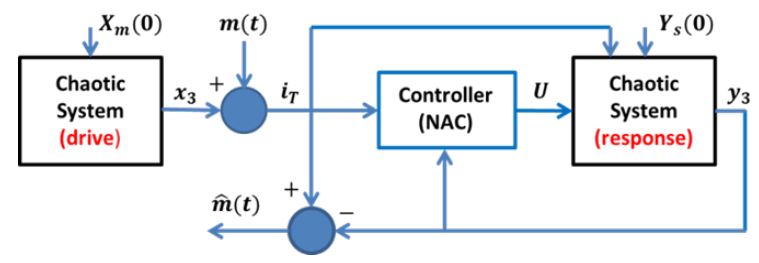

Fig 19. Transmitter-Receiver setup for secure communication.

The proposed synchronization method enhances the security of the transmitted signal $\left(i_{T}\right)$. The original and recovered message signals are shown in Figure 20, which reveals that synchronization is by using a single driving signal in secure communication. Using enhanced chaotic behavior of the proposed chaotic system enables difficulty for intruder large bandwidth of chaotic signal facilitates more powerful encryption of the information or message signal during communication.
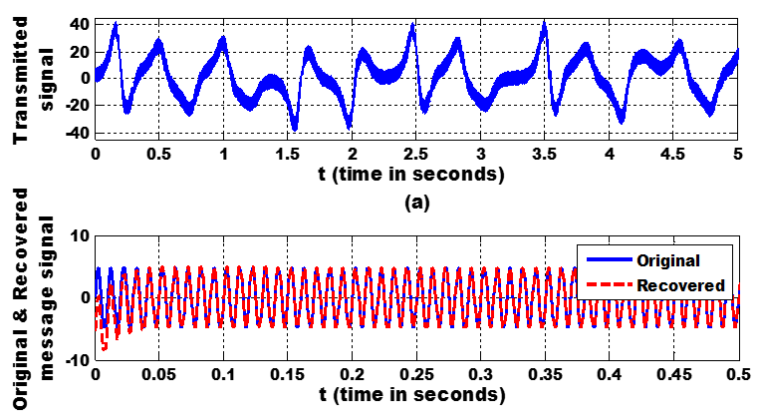

(b)

Fig 20. Shows chaotic secure communication, (a) Information bearing signal as transmitted signal, and (b) original and recovered message signals in blue and red colours, respectively. 


\section{Conclusion and Future Scope}

In this paper, a novel 3D chaotic system with saddle focus equilibria is analyzed. The system has chaotic and limit cycle behaviors. The dynamical behaviors are analyzed using different tools such as calculation of divergence, Lyapunov exponents and dimension, Lyapunov spectrum, basin of attraction, recurrence plot, Poincare map and frequency spectrum which illustrate that the proposed system has rich chaotic behaviors. The first positive LE (2.9733) implies chaos behavior, and its high value reflects more chaotic nature. The frequency spectrum of proposed system has a significantly broad frequency range i.e., large bandwidth $(\approx 24 \mathrm{~Hz})$ than six well-known chaotic systems. The system has basin of chaotic attractors for which first Lyapunov exponent ranges between 2.5 to 3 . Such unique behaviours arise form a $3 \mathrm{D}$ chaotic system with saddle focus equilibria is rare in the literature. MATLAB simulations are used to verify theoretical analyses. Further, an analog circuit is designed to realize the proposed chaotic system and its synchronization. The circuit simulation results correspond the obtained MATLAB simulation results. Analog circuit simulation results have good qualitative agreement. Large bandwidth enabled more secure encryption of the information signal during communication to achieve high-performance, secure communication and demonstrated in application to secure communication.

Moreover, the designed control technique is not robust against the parametric uncertainties and external disturbances, reflects its limitation and design of a robust nonlinear active control technique is challenging task and may be explored by researchers in future. Further, communication security in the complex dynamical communication networks is the dire need and trending research nowadays. The proposed wide band chaotic system, its synchronisation may help in complex dynamical communication networks and still needs to be investigated.

\section{Acknowledgement}

The research work is supported by the TEQIP-III Minor Research Grant, National Institute of Technology Meghalaya, India. Sanctioned Project Ref. No. NITMGH/TEQIP-III/MP/2019-20/269.

\section{References}

1) Volkovskii AR, Tsimring L, Rulkov SNF, Langmore L. and Langmore I. Spread spectrum communication system with chaotic frequency modulation. An Interdisciplinary Journal of Nonlinear Science. 2005;15. Available from: https://doi.org/10.1063/1.1942327.

2) Nuñez-Perez JC, Adeyemi VA, Sandoval-Ibarra Y, Pérez-Pinal FJ, Tlelo-Cuautle E. FPGA Realization of Spherical Chaotic System with Application in Image Transmission. Mathematical Problems in Engineering. 2021;2021:Article ID 5532106. Available from: https://doi.org/10.1155/2021/5532106.

3) Marek B. Chaos Predictability in a Chemical Reactor. International Journal of Bifurcation and Chaos. 2020;30(11):2050221. Available from: https: //doi.org/10.1142/S0218127420502211.

4) Saheb P, Mainul H, Pijush P, Pati NC, Pal N, Joydev C. Cooperation delay induced chaos in an ecological system. An Interdisciplinary Journal of Nonlinear Science. 2020;30(8):83124. Available from: https://doi.org/10.1063/5.0012880.

5) Sahin ME, Taskiran ZGC, Guler H, Hamamci SE. Application and Modeling of a Novel 4D Memristive Chaotic System for Communication Systems. Circuits, Systems, and Signal Processing. 2020;39:3320-3349. Available from: https://doi.org/10.1007/s00034-019-01332-6.

6) Singh PP, Roy BK. Inter network synchronization of complex dynamical networks by using smooth proportional integral SMC technique. European Physics Journal Special Topics. 2020;229:861-876. Available from: https://doi.org/10.1140/epjst/e2020-900149-3.

7) Edward NL. Deterministic nonperiodic flow. Journal of the Atmospheric Sciences. 1963;20:130-141. Available from: https://doi.org/10.1175/15200469(1963)020<0130:DNF>2.0.CO;2.

8) Rossler OE. An equation for continuous chaos. Physics Letters A. 1976;57(5):90101-90109. Available from: https://doi.org/10.1016/0375-9601(76)901018.

9) Chen $\mathrm{CH}$, Sheu LJ, Chen HK, Chen JH, Wang HC, Chao YC, et al. A novel hyper-chaotic system and its synchronization. Nonlinear Analysis: Real World Applications. 2009;10(4):2088-2096. Available from: https://doi.org/10.1016/j.nonrwa.2008.03.015.

10) Julien CS. Chaos and Time-Series Analysis. London. Oxford University Press. 2003. Available from: http://sprott.physics.wisc.edu/chaostsa/.

11) Chongxin L, Tao LT, Ling L, Kai L. A novel chaotic attractor. Chaos, Solitons and Fractals. 2004;22(5):1031-1038. Available from: https://doi.org/10.1016/ j.chaos.2007.04.025.

12) Jinhu L, Guanrong C. A novel chaotic attractor coined. International Journal of Bifurcation and Chaos. 2002;12(3):659-661. Available from: https: //doi.org/10.1142/S0218127402004620.

13) Wenbo L, Guanrong C. A novel chaotic system and its generation. International Journal of Bifurcation Chaos. 2003;13(1):261-267. Available from: https://doi.org/10.1142/S0218127403006509.

14) Singh PP, Roy BK. A novel chaotic system without equilibria, with parachute and thumb shapes of Poincare map and its projective synchronization. European Physics Journal Special Topics. 2020;229:1265-1278. Available from: https://doi.org/10.1140/epjst/e2020-900259-0.

15) Guoyuan Q, Guanrong C, Yuhui Z. On a novel asymmetric chaotic system. Chaos Solitons and Fractals. 2008;37:409-423. Available from: https: //doi.org/10.1016/j.chaos.2006.09.012.

16) Ihsan P, Yilmaz U. A novel chaotic attractor from general Lorenz system family and its electronic experimental implementation. Turkish Journal of Electrical Engineering and Computer Sciences. 2010;18(2).

17) Singh PP, Roy BK. Comparative performances of synchronization between different classes of chaotic systems using three control techniques. Annual Reviews in Control. 2018;45(3):152-165. Available from: https://doi.org/10.1016/j.arcontrol.2018.03.003.

18) Ihsan P, Yllmaz U. A novel 3D chaotic system with golden proportion equilibria: Analysis and electronic circuit realization. Computers and Electrical Engineering. 2012;38:1777-1784. Available from: https://doi.org/10.1016/j.compeleceng.2012.08.007. 
19) Vaidyanathan S, Ihsan P. Analysis, control, synchronization, and circuit design of a novel chaotic system. Mathematical and Computer Modelling. 2012;55:1904-1915. Available from: https://doi.org/10.1016/j.mcm.2011.11.048.

20) Singh PP, Roy BK. Memristor-based novel complex-valued chaotic system and its projective synchronization using nonlinear active control technique. European Physics Journal Special Topics. 2019;228:2197-2214. Available from: https://doi.org/10.1140/epjst/e2019-900036-5.

21) Abooee A, Yaghini-Bonabi HA, Jahed-Motlagh MR. Analysis and circuitry realization of a novel three-dimensional chaotic system. Communications in Nonlinear Science and Numerical Simulation. 2013;18:1235-1245. Available from: https://doi.org/10.1016/j.cnsns.2012.08.036.

22) Yongjian L. Circuit implementation and finite-time synchronization of the $4 \mathrm{D}$ Rabinovich hyperchaotic system. Nonlinear Dynamics. 2012;67:89-96. Available from: https://doi.org/10.1007/s11071-011-9960-2.

23) Ahmed GR, Ahmed MS, Abdel-Latif ES. MOS realization of the double-scroll-like chaotic equation. IEEE Transactions on Circuits and Systems-I: Fundamental Theory and Applications. 2003;50(2):285-288. Available from: https://doi.org/10.1109/TCSI.2002.808217.

24) Louis MP, Thomas LC. Synchronization of chaotic systems. Chaos: An Interdisciplinary Journal of Nonlinear Science. 2015;25:97611. Available from: https://doi.org/10.1063/1.4917383.

25) Singh PP, Singh JP, Roy BK. Synchronization and anti-synchronization of Lu and Bhalekar-Gejji chaotic systems using nonlinear active control. Chaos, Solitons and Fractals. 2014;69:31-39. Available from: https://doi.org/10.1016/j.chaos.2014.09.005.

26) Vaidyanathan $S$. Adaptive design of controller and synchronizer for Lu-Xiao chaotic system with unknown parameters. International Journal on Computer Science and Information Technology. 2013;5:197-210. Available from: https://doi.org/10.5121/IJCSIT.2013.5116.

27) Singh PP, Singh KM, Roy BK. Chaos control in biological system using recursive backstepping sliding mode control. European Physics Journal Special Topics. 2018;227:731-746. Available from: https://doi.org/10.1140/epjst/e2018-800023-6.

28) Junchan Z, Jun-An L. Using sampled-data feedback control and linear feedback synchronization in a novel hyperchaotic system. Chaos, Solitons and Fractals. 2008;35:376-382. Available from: https://doi.org/10.1016/j.chaos.2006.05.035.

29) Hongmei G, Shouming Z. Synchronization criteria of time-delay feedback control system with sector bounded nonlinearity. Applied Mathematics and Computation. 2009;191:550-557. Available from: https://doi.org/10.1016/j.amc.2007.02.154.

30) Singh PP, Roy BK. Microscopic chaos control of chemical reactor system using nonlinear active plus proportional integral sliding mode control technique. European Physics Journal Special Topics. 2019;228:169-184. Available from: https://doi.org/10.1140/epjst/e2019-800115-4.

31) Faqiang W, Chongxin L. Synchronization of unified chaotic system based on passive control. Physics D: Applied Physics. 2007;225:55-60. Available from: https://doi.org/10.1016/j.physd.2006.09.038.

32) Yu-Ping T, Xinghuo Y. Stabilizing unstable periodic orbits of chaotic systems via an optimal principle. Journal of Franklin Institute. 2000;337:47-55. Available from: https://doi.org/10.1016/S0016-0032(00)00047-8.

33) Jae-Hun K, Chang-Woo P, Euntai K, Mignon P. Fuzzy adaptive synchronization of uncertain chaotic systems. Physics Letters A. 2004;334:295-305. Available from: https://doi.org/10.1016/j.physleta.2004.11.033.

34) Coelho LDS, Bernert DLDA. PID control design for chaotic synchronization using a Tribes optimization approach. Chaos, Solitons and Fractals. 2009;42:634-640. Available from: https://doi.org/10.1016/j.chaos.2009.01.032.

35) Bernardini D, Grzegorz L. An overview of 0-1 test for chaos. Journal of the Brazilian Society of Mechanical Sciences and Engineering. 2016;38(5):1433-1450. Available from: https://doi.org/10.1007/s40430-015-0453-y.

36) Sabarathinam S, K T. Transient chaos in a globally coupled system of nearly conservative Hamiltonian Duffing oscillators. Chaos, Solitons and Fractals. 2015;73:129-140. Available from: https://doi.org/10.1016/j.chaos.2015.01.004.

37) Norbert M, Romano MC, Thiel M, Kurths J. Recurrence Plots for the Analysis of Complex Systems. Physics Reports. 2007;438(5-6):237-329. Available from: https://doi.org/10.1016/j.physrep.2006.11.001.

38) Iftikhar A, Chunlai M, Fuchen Z. A novel chaotic attractor with quadratic exponential nonlinear term from Chen's attractor. International Journal of Analysis and Applications. 2014;5(1):27-32. Available from: http://etamaths.com/index.php/ijaa/article/view/197.

39) Fa-Qiang W, Chong-Xin L. Hyperchaos evolved from the Liu chaotic system. Chinese Physics. 2006;15(5):963-966. Available from: https://doi.org/10. 1088/1009-1963/15/5/016.

40) Bruce AR, Stéphane M, Pier M, Erik B. Nonlinear characterization of a Rossler system under periodic closed-loop control via time-frequency and bispectral analysis. Mechanical Systems and Signal Processing. 2018;99:567-585. Available from: https://doi.org/10.1016/j.ymssp.2017.06.001. 